\title{
Estudio comparativo de la Frecuencia Fundamental, Jitter y Shimmer en escolares normo oyentes e hipoacúsicos
}

\section{Comparative study of the Fundamental Frequency, Jitter and Shimmer in normal hearing and hard of hearing schoolchildren}

\author{
Silvia Tolosa-López ${ }^{1}$, Nora Crespo-Pérez ${ }^{2}$, Andrés Llanos-Redondo ${ }^{2}$, Sandra Aguilar-Cañas ${ }^{2}$, \\ Heriberto Rangel-Navia ${ }^{2}$, Edwin Mauricio Portilla-Portilla ${ }^{2}$ Diego Rivera-Porras ${ }^{3 *}$, \\ 1 IPS Fomesalud, Colombia. \\ 2 Universidad de Pamplona, Colombia. \\ 3 Universidad Simón Bolívar, Colombia.
}

*Dirigir correspondencia a: $\underline{\text { d.rivera@unisimonbolivar.edu.co }}$

\begin{tabular}{|c|c|}
\hline \multirow[b]{3}{*}{ Article History } & \multirow{7}{*}{$\begin{array}{l}\text { RESUMEN } \\
\text { Introducción: La función vocal depende el feedback auditivo en este sentido análisis de las } \\
\text { variaciones en la frecuencia fundamental y las perturbaciones de la voz en escolares con } \\
\text { hipoacusia y normo oyentes permite establecer parámetros de referencias de duración en la } \\
\text { transición entre sílabas, tensión, desequilibrio de resonancia, alta frecuencia, dificultades en la } \\
\text { coordinación fonorespiratoria y frecuente incoordinación fonatoria con una variación excesiva. } \\
\text { Métodos: Estudio cuantitativo descriptivo de alcance comparativo entre los parámetros acústicos } \\
\text { de: frecuencia fundamental (FO), Jitter y Shimmer, de } 38 \text { escolares, de los cuales } 19 \text { Hipoacusia } \\
\text { bilateral y } 19 \text { niños con audición normal, en edades comprendidas entre } 9 \text { a } 15 \text { años, habitantes } \\
\text { de la ciudad de Cúcuta, Norte de Santander. Resultados: Uno de los escolares del grupo de } \\
\text { audición normal presento tapón parcial unilateral que no afecto el umbral auditivo, en relación al } \\
\text { tipo de hipoacusia } 17 \text { con hipoacusia neurosensorial y } 2 \text { con hipoacusia conductiva con grados de } \\
\text { moderado a profunda, se encontró asociaciones significaras en variaciones de las perturbaciones } \\
\text { en donde el JITTER em mayo en los escolares con hipoacusias y el SHIMMER en mayor en } \\
\text { población normvo oyente Conclusiones: La hipoacusia, se presenta una inadecuada producción } \\
\text { del habla unida a una deficiente comprensión del lenguaje, debido a la incorrecta realización del } \\
\text { fenómeno de feedback, será muy difícil la autocorrección de sus dificultades articulatorias. }\end{array}$} \\
\hline & \\
\hline & \\
\hline Received: 041021 & \\
\hline :29 1121 & \\
\hline Publist & \\
\hline & \\
\hline
\end{tabular}

Palabras clave: Acústica del Lenguaje; voz; calidad de la voz; pérdida auditiva; fonación.

DOI 10.17081/innosa.143

(C)Copyright 2021 Tolosa-

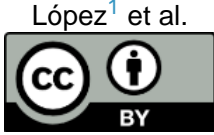

\section{ABSTRACT}

Background: The vocal function depends on the auditory feedback in this sense. Analysis of the variations in the fundamental frequency and the disturbances of the voice in schoolchildren with hearing loss and normal hearing allows to establish reference parameters of duration in the transition between syllables, tension, resonance imbalance, high frequency, difficulties in speechbreathing coordination and frequent speech incoordination with excessive variation. Methods: Quantitative descriptive study of comparative scope between the acoustic parameters of: fundamental frequency (FO), Jitter and Shimmer, of 38 schoolchildren, of which 19 bilateral hearing loss and 19 children with normal hearing, aged between 9 to 15 years, inhabitants of the city of Cúcuta Norte de Santander. Results: One of the schoolchildren in the normal hearing group presented a unilateral partial plug that did not affect the hearing threshold, in relation to the type of hearing loss 17 with sensorineural hearing loss and 2 with conductive hearing loss with degrees of moderate to profound, significant associations were found in variations of the disturbances where the JITTER in May in schoolchildren with hearing loss and the SHIMMER in greater in the normal hearing population. Conclusions: Hearing loss, inadequate speech production together with poor language comprehension, due to the incorrect performance of the feedback phenomenon, will be very difficult to self-correct their articulatory difficulties.

Keywords: Acoustics of language; voice; voice quality; hearing loss; phonation.

Como citar: Tolosa-López S, Crespo-Pérez N , Llanos-Redondo A, Aguilar-Cañas S, Rangel-Navia H, Portilla-Portilla E, RiveraPorras D. Estudio comparativo de la Frecuencia Fundamental, Jitter y Shimmer en escolares normo oyentes e hipoacúsicos. Ciencia e Innovación en Salud. 2021. E142: 325-340 DOI 10.17081/innosa.143

Cite this dataset: Innovación en Salud, Ciencia e (2021), "Comparative study of the Fundamental Frequency, Jitter and Shimmer in normal hearing and hard of hearing schoolchildren", Mendeley Data, V1, doi: 10.17632/jfz77j6kwx.1 


\section{INTRODUCCIÓN}

El control nervioso de la producción vocal, está regulado por la zona cerebral motora de la faringolaringe y la parte posterior de la primera circunvolución frontal, extendida hacia el área motora suplementaria, así mismo, el control de los centros auditivos juega un papel muy importante, considerando la producción vocal como aquel resultado de la coordinación neuromuscular de los órganos involucrados en el acto fonatorio, incluyendo tanto los músculos de la postura y de la respiración, como los músculos de la laringe y el aparato fonoarticulatorio(1)(2)(3)(4).

El acceso a los sonidos del habla durante los primeros meses de vida, es fundamental para el correcto desarrollo del lenguaje oral, las alteraciones auditivas durante este periodo asociado a la hipoacusia ocasionan secuelas irreversibles en el córtex auditivo cerebral(5)(6), siendo la hipoacusia un término general para describir la disminución de la capacidad auditiva(7)(8); Como consecuencia, los niños con una determinada deficiencia auditiva pueden enfrentarse a dificultades en el desarrollo de la producción del habla, dependiendo del grado de la afectación de la vía auditiva aunque se trate con una adaptación audio protésica o implante( $\underline{9}$.

El análisis de la voz de personas con discapacidad auditiva, resaltan que durante la producción oral presentan, una mayor duración en la transición entre sílabas (10), dificultad en la articulación de los fonemas que no se perciben mediante la lectura labial (4), un tono fundamental de la voz más agudo(11), incluyen tensión, desequilibrio de resonancia, alta frecuencia, dificultades en la coordinación fonorespiratoria con una variación excesiva $\underline{(12)(13)}$.

El análisis acústico se apoya en ciertas medidas de perturbación o de corto plazo, que se caracterizan por brindar una ratificación perceptual. Los parámetros obtenidos por el análisis acústico permiten describir la voz de manera objetiva, teniendo en cuenta, la existencia de bases de datos normativas que caracterizan la calidad de la voz o el uso de herramientas inteligentes, que combinan los diversos parámetros, es posible distinguir entre la voz normal y la patológica, o incluso identificar o sugerir la patología. Estas herramientas permiten el monitoreo del punto de vista clínico y / o el empleo y reducen el grado de subjetividad del análisis perceptivo $\underline{(11)}$.

El análisis de la perturbación se basa en la evidencia de que, en una señal de voz, siempre están presentes pequeñas variaciones en la frecuencia, amplitud y forma de onda. Para describir las características vocales, las mediciones de estas perturbaciones que se tienen en cuenta en esta investigación son, amplitud y la frecuencia (14).

De esta manera, se encuentra el tono que es una cualidad producida por la frecuencia vibratoria de los pliegues vocales, este permite distinguir cuándo un sonido es agudo y cuándo es grave. Es decir, a mayor frecuencia el tono será agudo y a menor frecuencia el sonido será grave. El Jitter, corresponde a una variación de la frecuencia fundamental ciclo a ciclo, relacionado con la variación en el corto plazo: el jitter mide cuánto difiere un período dado del período que lo sucede inmediatamente. Este puede alterarse por diferentes causas ya sean neurológicas, por falta de control del sistema nervioso sobre los músculos vocales, aerodinámicas, cuando existe un defecto de cierre glótico que pueda provocar escape de aire que haga vibrar irregularmente a las cuerdas y mecánicas, cuando hay asimetrías en la masa de las cuerdas o cuando hay cambios en las propiedades biomecánicas de las mismas.Por otra parte, tenemos la intensidad que distingue la fuerza con la que se emite el sonido, y depende exclusivamente de la amplitud de vibración de la onda, cuando existe una irregularidad de la misma se presenta lo que se cataloga como Shimmer; mide la perturbación de la amplitud ciclo a ciclo y está condicionada a la amplitud de la vibración de las cuerdas vocales y a la presión subglótica. Como se trata de parámetro de la intensidad se ve afectado por un soporte respiratorio inadecuado, un cierre glótico incompleto o unas cuerdas vocales poco flexibles. Lo que concierne a la duración; como su nombre lo indica es el tiempo que permanece esa sensación auditiva de la producción del sonido. Y por último el timbre, que ayuda a 
diferenciar la fuente origen de dicha producción verbal, de esta manera se pueden distinguir voces, ruidos e instrumentos diferentes $\underline{(11)(15)}$.

En este sentido el presente artículo responde a la pregunta ¿Cuál es la variabilidad de las medidas de perturbación de la voz en niños con discapacidad auditiva en relación a niños normo oyentes en edades comprendidas de 9 a 15 años?

\section{MÉTODOS}

Estudio cuantitativo descriptivo de alcance comparativo con muestreo dirigido dadas las características de la investigación (16) entre las cuales se resaltan los parámetros acústicos de: frecuencia fundamental (FO), Jitter y Shimmer, de 38 escolares, de los cuales 19 Hipoacusia bilateral y 19 niños con audición normal, en edades comprendidas entre 9 a 15 años, habitantes de la ciudad de Cúcuta, Norte de Santander.

Para la selección de los participantes los escolares tendrán que cumplir con los siguientes criterios:

2.1 Criterios de inclusión. Se tuvieron en cuenta escolares con edades de 9 y 15 años, que presenten discapacidad auditiva, escolares quienes sus padres o acudientes firmaron el consentimiento informado, escolares con previo examen de Otoscopia y Audiometría; a su vez, escolares con edades de 9 y 15 años, sin alteraciones auditivas y escolares con previo examen de Otoscopia y Audiometría

2.2. Criterios de exclusión: Niños que no diligenciaron el consentimiento informado, niños que no estuviesen dentro del rango de edad.

\subsection{Procedimientos}

A todos los participantes se les realizó:

Exámenes audiológicos

\subsubsection{Otoscopia}

Se realiza exploración o del oído externo y el tímpano con la ayuda de un otoscopio CIS- EMRI12671 -RICSO MARCA WELCH ALLYN a todos los participantes, con el fin de definir el carácter normal o patológico de la porción externa del oído (17).

\subsubsection{Audiometría}

Posteriormente, se realizó medición de la capacidad de cada oído con el fin percibir las vibraciones de diversas bandas del espectro audible, a través de un audiómetro MAICO MA 41, en cabina Sonoamortiguada SILVHER SP090 certificada en norma as normas ANSI S3.1e ISO 8253-1, para localizar el umbral auditivo del oído (18); utilizado y descartar algún tipo de alteración auditiva en los niños normoyentes; así mismo, clasificar el grado de pérdida auditiva de la población de niños con discapacidad auditiva.

\subsection{Recolección de las muestras de voz.}

Para este procedimiento se utilizó un micrófono unidireccional modelo SHURE SM86, con adaptador SHURE X2u, que se colocó a $17 \mathrm{~cm}$ de la boca de los participantes, mientras realizaban la fonación sostenida de las 5 vocales del español hablado, durante 10 segundos al interior de la cabina sonoamortiguada de marca SILVHER SP090 certificada en normas ANSI S3.1e ISO 8253. Las muestras de voz se preamplificó y digitalizó utilizando una interfaz de audio conectada al computador 
portátil de marca ASUS S 46C, con una tarjeta de sonido con resoluciones de 16 bits y frecuencias de muestreo de $44.100 \mathrm{~Hz}$.

Una vez que se tomaron las muestras se editaron a través del programa WaveSurfer Versión 1.8.5. La edición de cada sonido se realizó tomando el cuerpo de la emisión y desechando el ataque y la filatura de cada muestra. Las muestras se tomaron a una frecuencia e intensidad espontaneas.

\subsection{Procesamiento de los datos}

Para analizar las señales vocálicas se empleó la funcionalidad del Voice Report de PRAAT Doing Phonetics by Computer, versión 6.2.01, considerando los valores de la frecuencia fundamental (F0), Shimmer y Jitter, extraído del espectrograma de banda estrecha de cada una de las muestras de voz mediante el programa PRAAT (versión $6.2 .0 \underline{(19)(20)(21)}$.

\subsection{Análisis estadístico}

Para realizar el análisis de la información, en primera medida se organizaron los elementos y variables de estudio en una matriz de datos. Posteriormente se procesaron los datos a partir del software estadístico especializado PAST 3.0. y SPSS (versión 23.0).

El procedimiento consistió en 3 fases, en la primera se verificó la distribución de normalidad en los datos a través de la prueba de Shapiro-Wilk y se encontró que los mismos siguen una distribución libre $(p<0,05)$. Seguido a esto, se aplicó la prueba de hipótesis $U$ de Mann-Whitney para muestras independientes con el fin de verificar si las distribuciones de los datos son iguales al comparar las categorías de los grupos. Y finalmente, se calcularon los estadísticos descriptivos para observar en que variables se evidencian cambios significativos en las puntuaciones.

\section{RESULTADOS}

Del total de los 38 escolares que participaron se encontró:

Tabla 1. Datos sociodemográficos

\begin{tabular}{cccc}
\hline \multirow{2}{*}{ Demografía } & & \multicolumn{2}{c}{ Grupo } \\
& & Normoyentes & Hipoacusia \\
\hline & 9 & 0 & 1 \\
& 10 & 1 & 5 \\
& 11 & 8 & 3 \\
& 12 & 5 & 2 \\
& 13 & 4 & 4 \\
& 14 & 1 & 3 \\
& 15 & 0 & 1 \\
& Total & 19 & 19 \\
& Sexo & 11 & 11 \\
& Masculino & 8 & 8 \\
& Femenino & 19 & 19 \\
\hline
\end{tabular}

Fuente: Elaboración propia. 
Gráfico 1. Los 19 escolares con audición normal se encuentran entre los rangos de edad de 10 a 15 años.

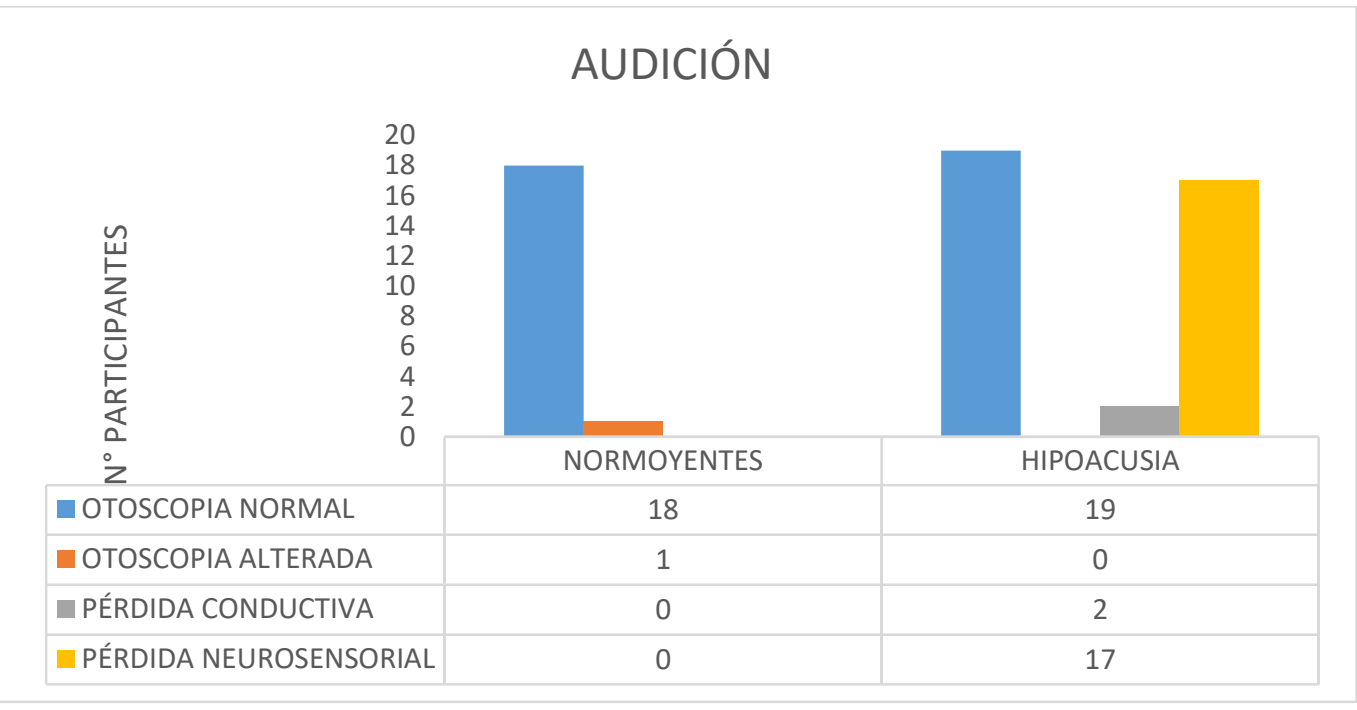

Fuente: Elaboración propia.

En los resultados de otoscopia se determina que dentro del grupo de escolares normoyentes 1 presenta tapón de cerumen parcial en oido derecho, que no afectó el umbral auditivo; en relación al tipo de hipoacusia de los 19 escolares posterior a la audiometría se confirmó el diagnóstico de 17 con hipoacusia neurosensorial y 2 con hipoacusia conductiva con grados de moderado a profunda (ver gráfica 1 y tabla 1).

Tabla 2. Análisis vocal /a/

\begin{tabular}{|c|c|c|c|c|c|c|c|c|c|}
\hline \multicolumn{4}{|c|}{ Resumen de prueba de hipótesis } & \multirow[b]{2}{*}{ /a/ } & \multirow{2}{*}{$\begin{array}{c}\text { Normoyentes } \\
\text { Media }\end{array}$} & \multirow{2}{*}{$\begin{array}{l}\text { Hipoacusia } \\
\text { Media }\end{array}$} & \multirow[b]{2}{*}{ Diferencia } & \multirow{2}{*}{$\begin{array}{c}\text { Diferencia } \\
\%\end{array}$} & \multirow{2}{*}{$\begin{array}{l}\text { Grupo que } \\
\text { predomina }\end{array}$} \\
\hline $\begin{array}{l}\text { Hipótesis } \\
\text { nula }\end{array}$ & Prueba & Sig. & Decisión & & & & & & \\
\hline $\begin{array}{l}\text { La } \\
\text { distribución } \\
\text { de F0 Hz-A es } \\
\text { la misma } \\
\text { entre las } \\
\text { categorías de } \\
\text { Grupo. }\end{array}$ & $\begin{array}{c}\text { U de } \\
\text { Mann- } \\
\text { Whitney }\end{array}$ & 0,01 & $\begin{array}{l}\text { Rechazar } \\
\text { la } \\
\text { hipótesis } \\
\text { nula. }\end{array}$ & $\mathrm{FO} \mathrm{Hz}$ & 206,88 & 257,37 & 50,49 & 19,62 & Hipoacusia \\
\hline $\begin{array}{l}\text { La } \\
\text { distribución } \\
\text { de JITTER } \\
\text { \%(LOCAL) es } \\
\text { la misma } \\
\text { entre las } \\
\text { categorías de } \\
\text { Grupo. }\end{array}$ & $\begin{array}{c}\text { U de } \\
\text { Mann- } \\
\text { Whitney }\end{array}$ & 0,00 & $\begin{array}{c}\text { Rechazar } \\
\text { la } \\
\text { hipótesis } \\
\text { nula. }\end{array}$ & $\begin{array}{c}\text { JITTER } \\
\%(\text { LOCAL) }\end{array}$ & 0,47 & 0,78 & 0,31 & 39,23 & Hipoacusia \\
\hline $\begin{array}{c}\text { La } \\
\text { distribución } \\
\text { de JITTER } \\
\text { (LOCAL, } \\
\text { ABSOLUTE) } \\
\text { SEG es la } \\
\text { misma entre } \\
\text { las categorías } \\
\text { de Grupo. }\end{array}$ & $\begin{array}{c}\text { Ude } \\
\text { Mann- } \\
\text { Whitney }\end{array}$ & 0,08 & $\begin{array}{l}\text { Retener la } \\
\text { hipótesis } \\
\text { nula. }\end{array}$ & $\begin{array}{l}\text { JITTER } \\
\text { (LOCAL, } \\
\text { ABSOLUTE) } \\
\text { SEG }\end{array}$ & 23,44 & 32,93 & & & \\
\hline $\begin{array}{c}\text { La } \\
\text { distribución } \\
\text { de SHIMMER } \\
\text { (LOCAL)\% es }\end{array}$ & $\begin{array}{c}\text { U de } \\
\text { Mann- } \\
\text { Whitney }\end{array}$ & 0,00 & $\begin{array}{l}\text { Rechazar } \\
\text { la } \\
\text { hipótesis } \\
\text { nula. }\end{array}$ & $\begin{array}{l}\text { SHIMMER } \\
\text { (LOCAL)\% }\end{array}$ & 6,91 & 4,00 & 2,91 & 42,08 & Normoyentes \\
\hline
\end{tabular}




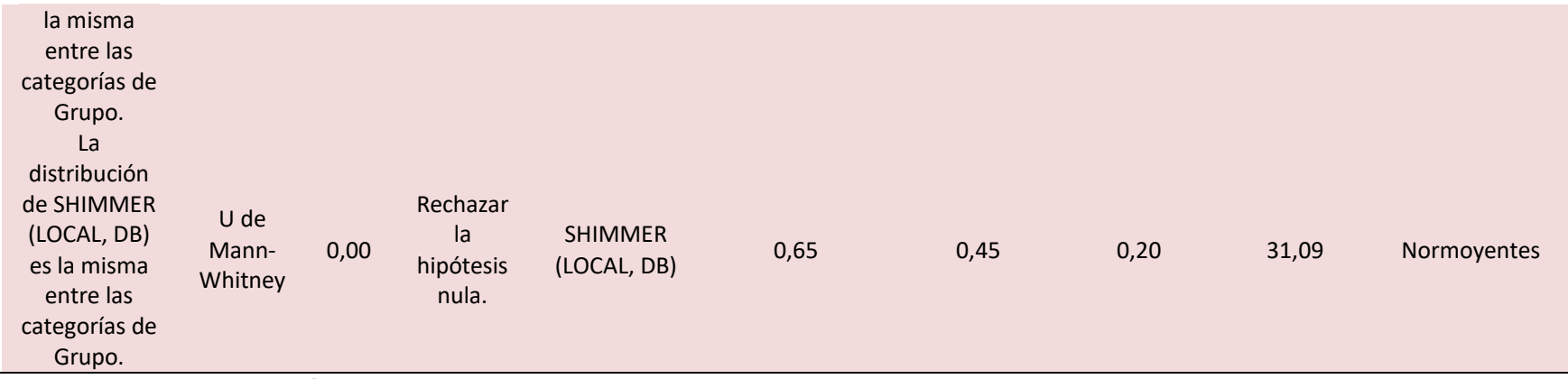

Fuente: Elaboración propia.

Se aplicó la prueba de hipótesis $U$ de Mann-Whitney para muestras independientes con el fin de identificar si existían diferencias estadísticamente significativas en las puntuaciones de las pruebas realizadas, y se encontró que si existen dichas diferencias $(p<0,05)$ en las variables $\mathrm{F} 0 \mathrm{~Hz}$, JITTER \%(LOCAL), JITTER (LOCAL ABSOLUTE) \%, SHIMMER (LOCAL)\%, SHIMMER (LOCAL, DB), Finalmente, al analizar de una forma más detallada, se encontró que la F0 en las dos poblaciones es de un 19,62\% siendo la población con hipoacusia quien presenta un aumento de la frecuencia debido al esfuerzo que deben realizar con relación a la vibración de los pliegues vocales (ver tabla 2).

Tabla 3. Análisis vocal /e/

\begin{tabular}{|c|c|c|c|c|c|c|c|c|c|}
\hline \multicolumn{4}{|c|}{ Resumen de prueba de hipótesis } & \multirow{2}{*}{ /e/ } & \multirow{2}{*}{$\begin{array}{c}\text { Normoyentes } \\
\text { Media }\end{array}$} & \multirow{2}{*}{$\begin{array}{c}\text { Hipoacusia } \\
\text { Media }\end{array}$} & \multirow{2}{*}{ Diferencia } & \multirow{2}{*}{ Diferencia \% } & \multirow{2}{*}{$\begin{array}{l}\text { Grupo que } \\
\text { predomina }\end{array}$} \\
\hline Hipótesis nula & Prueba & Sig. & Decisión & & & & & & \\
\hline $\begin{array}{l}\text { La distribución } \\
\text { de FO Hz es la } \\
\text { misma entre las } \\
\text { categorías de } \\
\text { Grupo. }\end{array}$ & $\begin{array}{c}\text { U de } \\
\text { Mann- } \\
\text { Whitney }\end{array}$ & 0,00 & $\begin{array}{c}\text { Rechazar } \\
\text { la } \\
\text { hipótesis } \\
\text { nula. }\end{array}$ & $\mathrm{FO} \mathrm{Hz}$ & 204,66 & 273,62 & 68,96 & 25,20 & Hipoacusia \\
\hline $\begin{array}{l}\text { La distribución } \\
\text { de JITTER } \\
\%(\text { LOCAL) es la } \\
\text { misma entre las } \\
\text { categorías de } \\
\text { Grupo. }\end{array}$ & $\begin{array}{c}\text { Ude } \\
\text { Mann- } \\
\text { Whitney }\end{array}$ & 0,01 & $\begin{array}{c}\text { Rechazar } \\
\text { la } \\
\text { hipótesis } \\
\text { nula. }\end{array}$ & $\begin{array}{c}\text { JITTER } \\
\%(\text { LOCAL } \\
\text { ) }\end{array}$ & 0,47 & 0,64 & 0,17 & 25,94 & Hipoacusia \\
\hline $\begin{array}{l}\text { La distribución } \\
\text { de JITTER } \\
\text { (LOCAL, } \\
\text { ABSOLUTE) SEG } \\
\text { es la misma } \\
\text { entre las } \\
\text { categorías de } \\
\text { Grupo. }\end{array}$ & $\begin{array}{c}\text { U de } \\
\text { Mann- } \\
\text { Whitney }\end{array}$ & 0,91 & $\begin{array}{c}\text { Retener } \\
\text { la } \\
\text { hipótesis } \\
\text { nula. }\end{array}$ & $\begin{array}{l}\text { JITTER } \\
\text { (LOCAL, } \\
\text { ABSOLUT } \\
\text { E) SEG }\end{array}$ & 25,31 & 24,02 & & & \\
\hline $\begin{array}{l}\text { La distribución } \\
\text { de SHIMMER } \\
\text { (LOCAL)\% es la } \\
\text { misma entre las } \\
\text { categorías de } \\
\text { Grupo. } \\
\text { La distribución } \\
\text { de SHIMMER } \\
\text { (LOCAL, DB) es } \\
\text { la misma entre } \\
\text { las categorías } \\
\text { de Grupo. }\end{array}$ & $\begin{array}{c}\text { Ude } \\
\text { Mann- } \\
\text { Whitney }\end{array}$ & 0,01 & $\begin{array}{c}\text { Rechazar } \\
\text { la } \\
\text { hipótesis } \\
\text { nula. }\end{array}$ & $\begin{array}{c}\text { SHIMME } \\
\text { R } \\
\text { (LOCAL) } \\
\%\end{array}$ & 0,54 & 0,36 & 0,19 & 34,42 & $\begin{array}{c}\text { Normoyente } \\
\mathrm{s}\end{array}$ \\
\hline
\end{tabular}

Fuente: Elaboración propia.

Con relación a la vocal /e/ la diferencia en cuanto a la frecuencia fundamental aumenta a 25,7\%, se puede corroborar en el resultado de perturbación Jitter (LOCAL) ya que su índice está por debajo de $1 \%$ correspondiente a la variación de la frecuencia fundamental entre un ciclo vocal (ver tabla 3 ). A 
su vez, el shimmer resulta en $2,48 \%$ en un rango de diferencia de $34,42 \%$. Generando mayor relevancia en la frecuencia de la vibración de las cuerdas aumentando así la F0.

Tabla 4. Análisis vocal /i/

\begin{tabular}{|c|c|c|c|c|c|c|c|c|c|}
\hline \multicolumn{4}{|c|}{ Resumen de prueba de hipótesis } & \multirow[b]{2}{*}{ /i/ } & \multirow{2}{*}{$\begin{array}{c}\text { Normoyentes } \\
\text { Media }\end{array}$} & \multirow{2}{*}{$\begin{array}{c}\text { Hipoacusia } \\
\text { Media }\end{array}$} & \multirow[b]{2}{*}{ Diferencia } & \multirow{2}{*}{$\begin{array}{c}\text { Diferencia } \\
\%\end{array}$} & \multirow{2}{*}{$\begin{array}{l}\text { Grupo que } \\
\text { predomina }\end{array}$} \\
\hline $\begin{array}{l}\text { Hipótesis } \\
\text { nula }\end{array}$ & Prueba & Sig. & Decisión & & & & & & \\
\hline $\begin{array}{c}\text { La } \\
\text { distribución } \\
\text { de FO Hz es la } \\
\text { misma entre } \\
\text { las categorías } \\
\text { de Grupo. }\end{array}$ & $\begin{array}{c}\text { Ude } \\
\text { Mann- } \\
\text { Whitney }\end{array}$ & 0,01 & $\begin{array}{c}\text { Rechazar } \\
\text { la } \\
\text { hipótesis } \\
\text { nula. }\end{array}$ & $\mathrm{FO} \mathrm{Hz}$ & 218,18 & 284,67 & 66,49 & 23,36 & Hipoacusia \\
\hline $\begin{array}{c}\text { La } \\
\text { distribución } \\
\text { de JITTER } \\
\%(\text { LOCAL) es } \\
\text { la misma } \\
\text { entre las } \\
\text { categorías de } \\
\text { Grupo. }\end{array}$ & $\begin{array}{c}\text { U de } \\
\text { Mann- } \\
\text { Whitney }\end{array}$ & 0,39 & $\begin{array}{c}\text { Retener la } \\
\text { hipótesis } \\
\text { nula. }\end{array}$ & $\begin{array}{c}\text { JITTER } \\
\%(\text { LOCAL) }\end{array}$ & 0,69 & 0,77 & & & \\
\hline $\begin{array}{c}\text { La } \\
\text { distribución } \\
\text { de JITTER } \\
\text { (LOCAL, } \\
\text { ABSOLUTE) } \\
\text { SEG es la } \\
\text { misma entre } \\
\text { las categorías } \\
\text { de Grupo. }\end{array}$ & $\begin{array}{c}\text { Ude } \\
\text { Mann- } \\
\text { Whitney }\end{array}$ & 0,51 & $\begin{array}{c}\text { Retener la } \\
\text { hipótesis } \\
\text { nula. }\end{array}$ & $\begin{array}{c}\text { JITTER } \\
\text { (LOCAL, } \\
\text { ABSOLUTE) } \\
\text { SEG }\end{array}$ & 32,04 & 27,73 & & & \\
\hline $\begin{array}{c}\text { La } \\
\text { distribución } \\
\text { de SHIMMER } \\
\text { (LOCAL)\% es } \\
\text { la misma } \\
\text { entre las } \\
\text { categorías de } \\
\text { Grupo. }\end{array}$ & $\begin{array}{c}\text { Ude } \\
\text { Mann- } \\
\text { Whitney }\end{array}$ & 0,00 & $\begin{array}{c}\text { Rechazar } \\
\text { la } \\
\text { hipótesis } \\
\text { nula. }\end{array}$ & $\begin{array}{l}\text { SHIMMER } \\
\text { (LOCAL)\% }\end{array}$ & 7,88 & 3,48 & 4,40 & 55,83 & Normoyentes \\
\hline $\begin{array}{c}\text { La } \\
\text { distribución } \\
\text { de SHIMMER } \\
\text { (LOCAL, DB) } \\
\text { es la misma } \\
\text { entre las } \\
\text { categorías de } \\
\text { Grupo. }\end{array}$ & $\begin{array}{c}\text { U de } \\
\text { Mann- } \\
\text { Whitney }\end{array}$ & 0,00 & $\begin{array}{c}\text { Rechazar } \\
\text { la } \\
\text { hipótesis } \\
\text { nula. }\end{array}$ & $\begin{array}{l}\text { SHIMMER } \\
\text { (LOCAL, DB) }\end{array}$ & 0,71 & 0,36 & 0,35 & 49,89 & Normoyentes \\
\hline
\end{tabular}

Fuente: Elaboración propia.

En el caso de la producción de la vocal/i/, la población con mayor siguen siendo los que presentan hipoacusia, se verifica el porcentaje de diferencia en la F0 y es de 23,36\%, permitiendo así confirmar aún más que la población requiere un aumento de la frecuencia de vibración de los pliegues vocales con relación a la población normoyente (ver tabla 4). En el caso de las perturbaciones no se observa alguna relevancia en el Jitter, pero en el caso del Shimmer el valor del Shimmer LOCAL presenta un porcentaje de 3, 48 lo que significa un aumento en la amplitud de ciclo vocal, lo que conlleva a requerir un aumento en la intensidad de producción de la vocal evaluada. 
Tabla 5. Análisis vocal /o/

\begin{tabular}{|c|c|c|c|c|c|c|c|c|c|}
\hline \multicolumn{4}{|c|}{ Resumen de prueba de hipótesis } & \multirow[b]{2}{*}{ /o/ } & \multirow{2}{*}{$\begin{array}{c}\text { Normoyentes } \\
\text { Media }\end{array}$} & \multirow{2}{*}{$\begin{array}{c}\text { Hipoacusia } \\
\text { Media }\end{array}$} & \multirow[b]{2}{*}{ Diferencia } & \multirow{2}{*}{$\begin{array}{c}\text { Diferencia } \\
\%\end{array}$} & \multirow{2}{*}{$\begin{array}{l}\text { Grupo que } \\
\text { predomina }\end{array}$} \\
\hline $\begin{array}{c}\text { Hipótesis } \\
\text { nula }\end{array}$ & Prueba & Sig. & Decisión & & & & & & \\
\hline $\begin{array}{c}\text { La } \\
\text { distribución } \\
\text { de F0 es la } \\
\text { misma entre } \\
\text { las categorías } \\
\text { de Grupo. }\end{array}$ & $\begin{array}{c}\text { Ude } \\
\text { Mann- } \\
\text { Whitney }\end{array}$ & 0,00 & $\begin{array}{l}\text { Rechazar } \\
\text { la } \\
\text { hipótesis } \\
\text { nula. }\end{array}$ & $\mathrm{FOHz}$ & 218,12 & 277,31 & 59,19 & 21,35 & Hipoacusia \\
\hline $\begin{array}{c}\text { La } \\
\text { distribución } \\
\text { de JITTER } \\
\%(\text { LOCAL) es } \\
\text { la misma } \\
\text { entre las } \\
\text { categorías de } \\
\text { Grupo. }\end{array}$ & $\begin{array}{c}\text { Ude } \\
\text { Mann- } \\
\text { Whitney }\end{array}$ & 0,19 & $\begin{array}{l}\text { Retener la } \\
\text { hipótesis } \\
\text { nula. }\end{array}$ & $\begin{array}{c}\text { JITTER } \\
\%(\text { LOCAL) }\end{array}$ & 0,51 & 0,72 & & & \\
\hline $\begin{array}{c}\text { La } \\
\text { distribución } \\
\text { de JITTER } \\
\text { (LOCAL, } \\
\text { ABSOLUTE) } \\
\text { SEG es la } \\
\text { misma entre } \\
\text { las categorías } \\
\text { de Grupo. }\end{array}$ & $\begin{array}{c}\text { Ude } \\
\text { Mann- } \\
\text { Whitney }\end{array}$ & 0,77 & $\begin{array}{l}\text { Retener la } \\
\text { hipótesis } \\
\text { nula. }\end{array}$ & $\begin{array}{c}\text { JITTER } \\
\text { (LOCAL, } \\
\text { ABSOLUTE) } \\
\text { SEG }\end{array}$ & 24,55 & 25,88 & & & \\
\hline $\begin{array}{c}\text { La } \\
\text { distribución } \\
\text { de SHIMMER } \\
\text { (LOCAL)\% es } \\
\text { la misma } \\
\text { entre las } \\
\text { categorías de } \\
\text { Grupo. }\end{array}$ & $\begin{array}{c}\text { Ude } \\
\text { Mann- } \\
\text { Whitney }\end{array}$ & 0,00 & $\begin{array}{l}\text { Rechazar } \\
\text { la } \\
\text { hipótesis } \\
\text { nula. }\end{array}$ & $\begin{array}{l}\text { SHIMMER } \\
\text { (LOCAL)\% }\end{array}$ & 6,61 & 3,21 & 3,40 & 51,44 & Normoyentes \\
\hline $\begin{array}{c}\text { La } \\
\text { distribución } \\
\text { de SHIMMER } \\
\text { (LOCAL, DB) } \\
\text { es la misma } \\
\text { entre las } \\
\text { categorías de } \\
\text { Grupo. } \\
\end{array}$ & $\begin{array}{c}\text { Ude } \\
\text { Mann- } \\
\text { Whitney }\end{array}$ & 0,00 & $\begin{array}{l}\text { Rechazar } \\
\text { la } \\
\text { hipótesis } \\
\text { nula. }\end{array}$ & $\begin{array}{l}\text { SHIMMER } \\
\text { (LOCAL, DB) }\end{array}$ & 0,62 & 0,33 & 0,29 & 47,08 & Normoyentes \\
\hline
\end{tabular}

Fuente: Elaboración propia.

Con relación a la muestra de la vocal /o/, los valores muestran relevancia de cambio en el Shimmer esto se debe a que cada vocal tiene una forma distinta en tracto vocal y por lo tanto valores formánticos distintos, hecho que permite diferenciar perceptualmente una vocal de otra, en el caso de la población con hipoacusia el shimmer muestra un valor de 3,21 lo cual supera el valor de índice en normalidad, lo que se confirma la necesidad de realizar una fuerza a la producción de dicha vocal (ver tabla 5).

Por último, en la producción de la vocal /u/, en la frecuencia fundamental tiene una diferencia de $24,18 \%$ evidenciando gran relevancia en el proceso de ubicación del tracto vocal y a su vez, se corrobora con el resultado del Jitter siendo este la perturbación generada en la frecuencia vibratoria de los pliegues vocales (ver tabla 6).

\subsection{ANÁLISIS.}

Cuantificar los parámetros acústicos de la voz e identificar y caracterizar los aspectos que afectan el comportamiento vocal de los escolares con hipoacusia que por ende, requieren de una intervención que prevenga futuras dificultades en la producción del lenguaje oral (11)(22); De esta manera, de acuerdo a los resultados obtenidos, se identificó a nivel general diferencias en el comportamiento en la 
frecuencia fundamental, y la perturbación Jitter y shimmer, durante la producción sostenida de la 5 vocales del español hablado.

Tabla 6. Análisis Vocal/u/

\begin{tabular}{|c|c|c|c|c|c|c|c|c|c|}
\hline \multicolumn{4}{|c|}{ Resumen de prueba de hipótesis } & \multirow{2}{*}{$/ \mathrm{u} /$} & \multirow{2}{*}{$\begin{array}{c}\text { Normoyentes } \\
\text { Media }\end{array}$} & \multirow{2}{*}{$\begin{array}{c}\text { Hipoacusia } \\
\text { Media }\end{array}$} & \multirow[b]{2}{*}{ Diferencia } & \multirow{2}{*}{$\begin{array}{c}\text { Diferencia } \\
\%\end{array}$} & \multirow{2}{*}{$\begin{array}{l}\text { Grupo que } \\
\text { predomina }\end{array}$} \\
\hline $\begin{array}{c}\text { Hipótesis } \\
\text { nula }\end{array}$ & Prueba & Sig. & Decisión & & & & & & \\
\hline $\begin{array}{c}\text { La } \\
\text { distribución } \\
\text { de F0 es la } \\
\text { misma entre } \\
\text { las categorías } \\
\text { de Grupo. }\end{array}$ & $\begin{array}{c}\text { U de } \\
\text { Mann- } \\
\text { Whitney }\end{array}$ & 0,00 & $\begin{array}{c}\text { Rechazar } \\
\text { la } \\
\text { hipótesis } \\
\text { nula. }\end{array}$ & $\mathrm{FO} \mathrm{Hz}$ & 215,36 & 284,02 & 68,67 & 24,18 & Hipoacusia \\
\hline $\begin{array}{c}\text { La } \\
\text { distribución } \\
\text { de JITTER } \\
\%(\text { LOCAL) es } \\
\text { la misma } \\
\text { entre las } \\
\text { categorías de } \\
\text { Grupo. } \\
\end{array}$ & $\begin{array}{c}\text { U de } \\
\text { Mann- } \\
\text { Whitney }\end{array}$ & 0,06 & $\begin{array}{l}\text { Retener la } \\
\text { hipótesis } \\
\text { nula. }\end{array}$ & $\begin{array}{c}\text { JITTER } \\
\%(\text { LOCAL) }\end{array}$ & 1,09 & 0,88 & & & \\
\hline $\begin{array}{c}\text { La } \\
\text { distribución } \\
\text { de JITTER } \\
\text { (LOCAL, } \\
\text { ABSOLUTE) } \\
\text { SEG es la } \\
\text { misma entre } \\
\text { las categorías } \\
\text { de Grupo. }\end{array}$ & $\begin{array}{c}\text { U de } \\
\text { Mann- } \\
\text { Whitney }\end{array}$ & 0,00 & $\begin{array}{c}\text { Rechazar } \\
\text { la } \\
\text { hipótesis } \\
\text { nula. }\end{array}$ & $\begin{array}{c}\text { JITTER } \\
\text { (LOCAL, } \\
\text { ABSOLUTE) } \\
\text { SEG }\end{array}$ & 54,61 & 31,57 & 23,04 & 42,19 & Normoyentes \\
\hline $\begin{array}{c}\text { La } \\
\text { distribución } \\
\text { de SHIMMER } \\
\text { (LOCAL)\% es } \\
\text { la misma } \\
\text { entre las } \\
\text { categorías de } \\
\text { Grupo. } \\
\end{array}$ & $\begin{array}{c}\text { U de } \\
\text { Mann- } \\
\text { Whitney }\end{array}$ & 0,00 & $\begin{array}{c}\text { Rechazar } \\
\text { la } \\
\text { hipótesis } \\
\text { nula. }\end{array}$ & $\begin{array}{l}\text { SHIMMER } \\
\text { (LOCAL)\% }\end{array}$ & 8,00 & 4,28 & 3,72 & 46,48 & Normoyentes \\
\hline $\begin{array}{c}\text { La } \\
\text { distribución } \\
\text { de SHIMMER } \\
\text { (LOCAL, DB) } \\
\text { es la misma } \\
\text { entre las } \\
\text { categorías de } \\
\text { Grupo. }\end{array}$ & $\begin{array}{c}\text { U de } \\
\text { Mann- } \\
\text { Whitney }\end{array}$ & 0,00 & $\begin{array}{c}\text { Rechazar } \\
\text { la } \\
\text { hipótesis } \\
\text { nula. }\end{array}$ & $\begin{array}{l}\text { SHIMMER } \\
\text { (LOCAL, DB) }\end{array}$ & 0,76 & 0,41 & 0,35 & 46,13 & Normoyentes \\
\hline
\end{tabular}

Fuente: Elaboración propia.

La perturbación de la frecuencia comúnmente denominada Jitter, se refiere a las variaciones involuntarias de la F0 que suceden de un ciclo a otro, representa también una medida de estabilidad de la fonación (23)(24)(25), de acuerdo a los resultados en la variable Jitter absoluto se evidenció para el sexo masculino no presenta diferencias significativas, las causas de las perturbaciones en la voz son diversas y se debe tener en cuenta al momento de interpretar los resultados, entre estas se encuentran las causas aerodinámicas, mecánicas y las neurológicas; estas últimas producen una gran variabilidad enJitter por falta de control del sistema nervioso sobre los músculos vocales (14)(26), en la edad de 12 a 14 años el valor de Jitter fue mayor en el grupo de escolares con hipoacusia para ambos sexos.

La variación de la amplitud de la onda de sonido es conocida como Shimmer, cambia con la reducción de la resistencia glótica o con la presencia de lesiones de masa en las cuerdas vocales y se correlaciona con la presencia de emisión de ruido, además está condicionada a la amplitud de la vibración de las cuerdas vocales y a la presión subglótica (27)(28)(29); Sin embargo, en esta variable los resultados de 
esta investigación no mostraron correlación teórica con respecto a la falta de retroalimentación auditiva al evidenciar que es mayor en el grupo de niños normo oyentes para ambos sexos, lo cual describe que la dificultad en el acceso auditivo al tratarse de la intensidad se ve afectada por un soporte respiratorio inadecuado o un cierre glótico incompleto(30)(31)(32).

Conocer los procesos fisiológicos que contribuyen al desarrollo vocal en niños con prótesis auditivas permite comprender las estrategias de producción del habla en esta población, tratando de establecer objetivos para una producción adecuada del habla. Es la retroalimentación auditiva la que regula la musculatura laríngea y las posturas del tracto vocal para una fonación adecuada (33), los formantes vocálicos son la cuantificación de las resonancias asociadas a la modificación de la forma del tracto vocal al momento de emitir una vocal, cuya representación gráfica se aprecia en cinco formantes (F1, F2, F3, F4 y F5) dentro de un espectrograma $(34)(35)(36)(37)$.

En relación a la evaluación de la estabilidad de la voz, mediante Jitter, Shimmer, se evidenció el grupo de niños con discapacidad auditiva, presentan mayor número de perturbaciones en la frecuencia, intensidad e irregularidad fonorespiratoria durante la producción vocal; a su vez, la falta de retroalimentación auditiva genera dificultad en la regulación neuromuscular del aparato fonador y la configuración del tracto vocal para la correcta producción vocal evidenciado a partir de los formantes F1 y F2.

\section{DISCUSIÓN}

El sistema auditivo es capaz de regular parámetros de la voz tales como la intensidad, frecuencia y posturas del tracto vocal en la fonación, en consecuencia, los trastornos vocales más comunes en los niños con discapacidad auditiva especialmente prelinguales, incluyen tensión, desequilibrio de resonancia, alta frecuencia, patrón de respiración alterado y expresión con variación excesiva, existe una falla en el monitoreo auditivo que son reflejadas en su producción vocal, porque además del control neuromuscular, la audición es de vital importancia para un buen desempeño en la comunicación oral (38)(39).

En este sentido, la frecuencia fundamental está determina por el total de ciclos glóticos en una unidad de tiempo (segundos); a su vez por factores como la longitud natural de las cuerdas vocales, estiramiento, la vibración y la tensión involucrada durante la producción vocal, se evidenció diferencias en la voz de los escolares con hipoacusia se presentó un mayor valor de fo en relación al grupo de niños normo oyentes, una frecuencia fundamental alta muestra un control laríngeo deficiente, también demostrado por la elevación de la laringe, un mayor esfuerzo de fonación y la incapacidad para controlar la tensión de las cuerdas vocales y la presión subglótica $\underline{(15)}$.

De acuerdo a lo anterior, es importante mencionar, que los seres humanos desde su nacimiento hasta la vejez se van transformando a nivel biopsicosocial y esto va de la mano de sus hábitos de vida saludable que pueden incidir de forma positiva o negativa en su evolución (40); Por su parte, la infancia es caracterizada por el crecimiento rápido y no homogéneo de las estructuras fonatorias, pues los tejidos laríngeos cambian de forma progresiva en tamaño, forma y posición, en consecuencia el acto fonatorio se modifica continuamente desde el nacimiento hasta la edad adulta, siendo los cambios mayormente marcados aquellos que suceden entre el nacimiento y la pubertad(41). Lo que explicaría porque en ciertas variables del estudio no hubo diferencias significativas entre ambas poblaciones, ya que, la voz de esta población con hipoacusia es resultado de dos factores relevantes como lo son la discapacidad auditiva y el estadio de edad en donde ocurre la también llamada muda vocal(42).

La muda vocal es un proceso que generalmente ocurre entre los 13 y 15 años; sin embargo, hay ocasiones en que el cambio de voz se retrasa o no se completa, lo cual, genera alteraciones denominadas trastornos de la muda vocal o puberfonías, caracterizada por una frecuencia fundamental más aguda que la correspondiente a la edad, además pueden existir bitonalidades, incoordinación fonorrespiratoria, aumento de tensión en la zona perilaríngea, se cree que la causa más común de este 
trastorno es la inadaptación muscular al cambio repentino que sufre el sistema fonatorio en esta $\operatorname{edad}(43)(44)$.

Estudios previos describen que a medida que el umbral auditivo empeora, la variabilidad de la frecuencia fundamental aumenta debido a una disminución en las señales auditivas para la correcta producción vocal (45); para este estudio el valor de la F0 es mayor en el grupo de escolares con hipoacusia; sin embargo, no se evidenciaron diferencias significativas entre sexos, es posible que la falta del feedback auditivo provoque un aumento de la tensión durante el ciclo glótico y una falta de control de la musculatura laríngea, lo que aumenta los valores de la frecuencia fundamental(45)(46).

Entre muchas otras características, diversos estudios frecuentemente refieren que las personas con discapacidad auditiva presentan voces agudas, siendo esta la correlación perceptiva de la frecuencia fundamental alta, que es causada por la falta de retroalimentación auditiva de la propia voz, como el control de la frecuencia, el cual se ve afectado cuando las funciones auditivas están deterioradas (47), así mismo, la intensidad es una variable medible que hace referencia a la presión subglótica espiratoria, una mayor contracción de los músculos involucrados en la respiración durante la emisión vocal, después del movimiento diafragmático durante la inspiración, significa un aumento del volumen o intensidad vocal (41). Como se evidenció en la voz del grupo de niños con discapacidad auditiva (casos) en la cual hubo una mayor intensidad durante la producción vocal tanto en el sexo femenino como en masculino en ambos rangos de edades.

\section{CONCLUSIONES}

En relación a la importancia teórica, la presente investigación proporciona información relevante sobre los marcadores acústicos de los sonidos vocálicos en niños con discapacidad auditiva con relación a niños normoyentes, información que puede servir de referencia para futuras investigaciones; además, contribuye en la generación de nuevo conocimiento dentro del campo fonoaudiológico.

Esta investigación demuestra a partir del análisis estadístico del comportamiento de las variables de perturbación de la amplitud de la voz Shimmer local\%, local, dB, apq3\% y apq5\%, entre los grupos de estudio, que al existir una discapacidad auditiva aumenta el número de perturbaciones en la fonación sostenida de las vocales.

Este estudio permitió caracterizar de manera completa la voz de niños con discapacidad auditiva y niños normo oyentes, en donde se identificó que existe una variabilidad estadísticamente significativa en los marcadores acústicos de la voz, generados principalmente por la falta del feedback auditivo; así mismo, se evidenció la influencia del sexo en la voz.

En la frecuencia fundamental de las edades estudiadas no hubo diferencias estadísticamente significativas en relación al sexo, sino al realizar la comparación entre niños con discapacidad auditiva y normo oyentes, lo cual resalta la importancia de la retroalimentación auditiva en la producción vocal, sin embargo, la intensidad vocal de los niños con discapacidad auditiva fue mayor para ambos sexos y grupo de edades.

Contribución de los autores/Author Contributions: la contribución de los autores corresponde; Conceptualización, todos; metodología, Diego Rivera Porras, Andrés Llanos Redondo; software, Diego Rivera Porras; validación, todos; análisis formal, todos; investigación, todos; recursos, todos.; curación de datos, Silvia Tolosa López, Nora Crespo Pérez; escritura: preparación del borrador original, todo; escritura: revisión y edición, todos; visualización, todos; supervisión, Heriberto Rangel Navia ,Edwin Mauricio Portilla Portilla6.; administración del proyecto, Heriberto Rangel Navia.; adquisición de fondos, Nora Crespo Pérez, Andrés Llanos Redondo, Sandra Aguilar Cañas ,Heriberto Rangel Navia. Todos los autores han leído y aceptado la versión publicada del manuscrito. 
Fondos: "Esta investigación fue financiada por la Universidad de Pamplona, con código 400-156.012068(GA317-BP-2017-FASEII)

Agradecimientos: Agradecemos al Departamento de Fonoaudiología de la Universidad de Pamplona Y el Programa de Psicología de la Universidad Simón Bolívar sede Cúcuta

Conflictos de intereses: Los autores declaran no tener ningún conflicto de intereses y los financiadores no tuvieron ningún rol en el diseño del estudio; en la recopilación, análisis o interpretación de datos; en la redacción del manuscrito o en la decisión de publicar los resultados.

\section{REFERENCIAS}

1. Giovanni A, Lagier A, Henrich N. Fisiología de la fonación. EMC - Otorrinolaringol. 2014 Aug 1; 43(3):1-16. DOl 10.1016/S1632-3475(14)68304-1

2. Eckland NS, Leyro TM, Mendes WB, Thompson RJ. The Role of Physiology and Voice in Emotion Perception During Social Stress. J Nonverbal Behav. 2019 Dec 1; 43(4):493-511. DOl $\underline{10.1007 / \mathrm{s} 10919-019-00311-4}$

3. Sjerps MJ, Fox NP, Johnson K, Chang EF. Speaker-normalized sound representations in the human auditory cortex. Nat Commun. 2019 Dec 1;10 (1). DOI 10.1038/s41467-019-10365-z

4. Núñez-Batalla F, Vasile G, Cartón-Corona N, Pedregal-Mallo D, Menéndez de Castro M, Guntín M, Gómez-Martínez J, Carro P, Llorente-Pendás J. Comparación de la producción vocal en niños con hipoacusia prelocutiva con adaptación audioprotésica e implante coclear con normooyentes. Acta Otorrinolaringol Esp. 2019; 70(5):251-7. DOI 10.1016/j.otorri.2018.05.004

5. Cordero Zanabria A, Jara Flores SGI. Comparación de formantes vocálicos en niños con pérdida auditiva usuarios de implante coclear y audífonos, y niños oyentes de 8 años de colegios de Surco [tesis de Maestría]. Perú: Pontif Univ Católica del Perú; 2016 Oct 20. Available from: http://tesis.pucp.edu.pe/repositorio/handle/123456789/7360

6. Jackson Menaldi, M. La voz normal y patológica [Internet]. 2019 [cited 2019 Nov 29]. 5-11 p. Available from: https://www.medicapanamericana.com/VisorEbookV2/Ebook/9789500695954\#\%7B\%22Pagina\%2 2:\%22Tapa\%22,\%22Vista\%22:\%22Indice\%22,\%22Busqueda\%22:\%22\%22\%7D

7. Rodríguez Hernández Y, Jiménez GER. Digital literacy in phonoaudiology: challenges of professional training since technological advances. Vol. 39, Revista de Logopedia, Foniatria y Audiologia. Grupo Ars XXI de Comunicacion, S.L.; 2019. p. 192-200.

8. Mendes R, Reis D, Marques Da Costa F, Carneiro A, Vieira MA. O papel do fonoaudiólogo frente a alterações fonoaudiológicas de audição, equilíbrio, voz e deglutição: uma revisão de literatura. Rev. CEFAC. 2015; 17(1):270-276. DOI 10.1590/1982-021620158414

9. Martínez C, Karol E, Castillo M, Oyarce CV, Romero LR, López I. Instrumento para evaluar las percepciones de las nociones de eficacia, eficiencia y efectividad terapéuticas utilizadas en la intervención fonoaudiológica de voz Universidad de Chile facultad de medicina escuela de fonoaudiología. 2014;1-24.

10. Ferreira J, Pérez G, Rangel H, Rivera D. Estrategias de intervención en el desarrollo de las habilidades comunicativas en personas en condición discapacidad: Una revisión sistemática. Gac Méd Caracas. 2021;129(1):107-127. 
11. Droguett YG. Aplicaciones clínicas del análisis acústico de la voz. Rev Otorrinolaringol y cirugía cabeza y cuello. 2017 Dec;77(4):474-83.

12. Martínez-Sánchez F, García-Melilán J, Carro J, Pujante I. Análisis acústico de las características espectrales de la expresión emocional de la voz. En: A. Acosta, J. L. Megías y J. Lupiáñez (Eds.), Avances en el estudio de la Motivación y la Emoción. Granada: AME; 2014. p. 6-12

13. Núñez Batalla F, González Márquez R, Peláez González MB, González Laborda I, Fernández Fernández M, Morato Galán M. Acoustic Voice Analysis Using the Praat programme: Comparative Study With the Dr. Speech Programme. Acta Otorrinolaringol (English Ed [Internet]. 2014 May 1 [cited 2018 Dec 1];65(3):170-6. DOl:10.1016/i.otorri.2013.12.004

14. Araújo SA, Grellet M, Pereira JC, Rosa MO. Normatização de medidas acústicas da voz normal. Rev Bras Otorrinolaringol. 2005;68(4):540-4. DOI 10.1590/S0034-72992002000400014

15. Delgado J, Nieves L, Jiménez A, Izquierdo L. Análisis acústico de la voz: medidas temporales, espectrales y cepstrales en la voz normal con el Praat en una muestra de hablantes de español. Rev. Investig. Logop. 2017; 7(2):108-27. Available from: https://dialnet.unirioja.es/servlet/articulo?codigo=6235178

16. Hernández R, et al. Metodología de la Investigación. 2a․ ed.McGraw-Hill. México, D.F., 2001. Pág. 52 - 134. Estaba

17. Flores Carmona E, Contreras Molina P, Sánchez P. Exploración fisíca del oído [Internet]. Málaga; 2015 [cited 2018 Nov 12]. Available from: https://seorl.net/PDF/Otologia/006\%20\%20EXPLORACI\%C3\%93N\%20F\%C3\%8DSICA\%20DEL\%20OIDO.pdf

18. García-Valdecasas J, Cardenete G, Zenker F. Guía de Práctica Clínica de Audiometría Tonal por Vía Aérea y Ósea con y sin Enmascaramiento [Internet]. 1st ed. AEDA Asociación Española de Audiología, editor. España: Revista electrónica de audiología; 2017 [cited 2018 Nov 12]. 74-87. DOI $\underline{10.51445 / \text { sja.auditio.vol4.2018.0059 }}$

19. Tuta E, Briceño I, Martínez J, Barrera S, Suarez V, Cardozo O. Utopía del tratamiento médico de la sordera: un agente cerumenolítico en el período colonial. Gac Méd Caracas 2021; 129(2):482-487. DOI: DOI 10.47307/GMC.2021.129.2.23

20. Elisei N. Acoustic analysis of normal and pathological voices using two different systems: anagraf and praat. Rev Psicol y Ciencias Afines [Internet]. 2012 [cited 2018 Dec 1]; 29(2):339-57. Available from: http://www.redalyc.org/articulo.oa?id=18026361002

21. Correa Duarte JA. Manual de análisis acústico del habla con Praat. Bogotá: Instituto Caro y Cuervo. Imprenta Patriótica; 2014.

22. Sales NJ, Queiroz Gurgel R, Rebelo Gonçalves MI, Côrtes Gama AC, Oliveira Cunha E, PradoBarreto VM, et al. Voice performance evaluation of street children from Aracaju, Brazil using perceptual-auditory and acoustic analyses. J Voice. 2014 Sep;27(5):589-94. DOI: 10.1016/j.jvoice.2013.02.009

23. Teixeira JP, Gonçalves A. Algorithm for Jitter and Shimmer Measurement in Pathologic Voices. Procedia Comput Sci [Internet]. 2016 Jan 1 [cited 2018 Dec 2];100:271-9. DOI $\underline{10.1016 / \text { j.procs.2016.09.155 }}$ 
24. Remacle A, Garnier M, Gerber S, David C, Petillon C. Vocal Change Patterns During a Teaching Day: Inter- and Intra-subject Variability. J Voice. 2018 Jan 1;32(1):57-63. DOI: 10.1016/i.jvoice.2017.03.008

25. Valero J, Gou J, Casanova C. Calidad vocal en niños sordos. La influencia del tipo de audífono empleado. Rev Logop Foniatría y Audiol [Internet]. 2006 Jul 1 [cited 2019 Jul 4];26(2):84-90. Available from: DOI:10.1016/S0214-4603(06)70106-1

26. Ramírez A, Martínez P, Cabrera J, Buestán P, Torracchi E, Carpio M. Habilidades sociales y agresividad en la infancia y adolescencia. AVFT. 2020; 39(2):209-14

27. Brockmann-Bauser M, Beyer D, Bohlender JE. Reliable acoustic measurements in children between 5;0 and 9;11 years: Gender, age, height and weight effects on fundamental frequency, jitter and shimmer in phonations without and with controlled voice SPL. Int J Pediatr Otorhinolaryngol. 2015 Dec 1; 79(12):2035-42. DOI: 10.1016/j.ijporl.2015.09.005

28. Spazzapan EA, Cardoso VM, Fabron EMG, Berti LC, Brasolotto AG, Marino VC de C. Acoustic characteristics of healthy voices of adults: From young to middle age. CODAS. 2018;30(5). DOI: $\underline{10.1590 / 2317-1782 / 20182017225}$

29. Lopes LW, Alves J do N, Evangelista D da S, França FP, Vieira VJD, de Lima-Silva MFB, et al. Accuracy of traditional and formant acoustic measurements in the evaluation of vocal quality. CODAS. 2018;30(5). DOI:10.1590/2317-1782/20182017282

30. Chou A, Schrof C, Polce E, Braden M, McMurray J, Jiang J. Comparing the Nonlinear Dynamic Acoustic Parameters of Healthy Adult and Pediatric Voices. Ann Otol Rhinol Laryngol. 2018 Dec 1;127(12):937-45. Doi: 0.1177/0003489418803394

31. Brockmann-Bauser M, Beyer D, Bohlender JE. Clinical relevance of speaking voice intensity effects on acoustic jitter and shimmer in children between 5;0 and 9;11 years. Int J Pediatr Otorhinolaryngol. 2014 Dec 1;78(12):2121-6. DOI: 10.1016/j.ijporl.2014.09.020

32. Vila-Rovira JM, Valero-Garcia J, Gonzalez-Sanvisens L. Indicadores fonorrespiratorios de normalidad y patología en la clínica vocal. Rev. investig. logop. 6 de abril de 2011. 2021 Dec 3; 1(1):35-55. DOl 10.5209/rlog.58706

33. Jiménez Romero MS. El impacto del implante coclear en la integración auditiva: resultados y factores predictores en un grupo de 116 niñas y niños sordos españoles. Rev Logop Foniatría y Audiol [Internet]. 2014 Jan [cited 2019 Jul 4];34(1):4-16. DOI 10.1016/i.rlfa.2013.06.001

34. Crespo N, Rivera E, Figueroa F, Chacón K, López L, Gonzalez S. Métodos de evaluación de la voz en docentes una revisión sistemática. Revista signos fónicos [Internet]. 2018 Jun 7 [cited 2019 Aug 2];3(1). Available http://revistas.unipamplona.edu.co/ojs_viceinves/index.php/CDH/article/view/2875

35. Terraza IG, Andonegi AR, Eta AEL, Ruíz UG. Diferencias en los formantes vocálicos de [a] según el tipo de emoción y otras variables sociolingüísticas. Bol Filol. 2015;50 (2):33-50.

36. Cordero Zanabria A, Jara Flores SGI. Comparación de formantes vocálicos en niños con pérdida auditiva usuarios de implante coclear y audífonos, y niños oyentes de 8 años de colegios de Surco. [cited $2019 \quad$ Nov 27];141. 2 Available http://tesis.pucp.edu.pe/repositorio/handle/20.500.12404/7360 
37. Lara Peinado A, Sistiaga Surez JA. Patología de la voz. Med - Programa Form Medica Contin Acreditado [Internet]. 2007 Nov [cited 2019 Jul 11];9(91):5876-84. DOI 10.1016/S02113449(07)74749-5.

38. Casanova Cori, JesúsValero Garcia JE. Calidad vocal de las personas con deficiencia auditiva a lo largo de la infancia y de la adolescencia. Rev Española Faniatría [Internet]. 2014 [cited 2018 Dec 7];3:12. Available from: https://www.researchgate.net/publication/281272713_La_calidad_vocal_de_las_personas_con_def iciencia_auditiva_a_lo_largo_de_la_infancia_y_de_la_adolescencia

39. Coelho AC, Brasolotto AG, Bevilacqua MC, Moret ALM, Júnior FB. Hearing performance and voice acoustics of cochlear implanted children. Braz J Otorhinolaryngol [Internet]. 2016 Jan [cited 2018 Nov 11]; 82(1):70-5. DOI: 0.1016/i.bjorl.2015.11.002

40. Nieto D, Nieto I. Estilo de vida de estudiantes de la Universidad del Atlántico: un estudio descriptivotransversal. Ciencia e Innovación en Salud. 2020. e68:19-30. DOl 10.17081/innosa.69

41. Ramírez L, Lizarazo Y, Bonilla N, Cardozo L, Cornejo A. Estrategias de intervención en la memoria de trabajo en niños y niñas sobrevivientes del desplazamiento forzado y la crisis fronteriza en Venezuela. AVFT. 2020; 39(3)

42. Chávez Muñoz SA, Peña Maldonado AC. Parámetros vocales y muda vocal en impedidos auditivos con implante coclear y audífonos. [Internet]. 2014. Available from: http://repositorio.unab.cl/xmlui/handle/ria/6356

43. Nercelles Carvajal L. Perfil vocal y psicosocial de los sujetos con puberfonía. Rev Logop Foniatr y Audiol. 2018 Apr 1;38(2):45-51. DOl 10.1016/j.rlfa.2017.06.001

44. Oliveira LF de, Corrêa C de C, Vieira MMRM, Blasca WQ, Brasolotto AG. Intervenção fonoaudiológica por meio da teleducação sobre a muda vocal e hábitos vocais. Audiol - Commun Res. 2018 Oct 11;23(0). DOI 10.1590/2317-6431-2017-1899

45. Hsu H-W, Fang T-J, Lee L-A, Tsou Y-T, Chen SH, Wu C-M. Multidimensional evaluation of vocal quality in children with cochlear implants: a cross-sectional, case-controlled study. Clin Otolaryngol [Internet]. 2014 Feb [cited 2019 Nov 27];39(1):32-8. DOI 10.1111/coa.12213

46. Mazzetto de Menezes KS, Master S, Guzman M, Bortnem C, Ramos LR. Diferencias de los parámetros acústicos y perceptuales de la voz entre sujetos ancianos y jóvenes con intensidad habitual y alta. Acta Otorrinolaringol Esp. 2014;65(2):76-84. DOI 10.1016/i.otorri.2013.07.009

47. Mahmoudi Z, Rahati S, Ghasemi MM, Asadpour V, Tayarani H, Rajati M. Classification of voice disorder in children with cochlear implantation and hearing aid using multiple classifier fusion. Biomed Eng Online. 2014 Jan 14;10:3-10. DOI: 10.1186/1475-925X-10-3 